REVIEW

\title{
Evolving immunotherapeutic strategies in bladder and renal cancer
}

\author{
T R L Griffiths, J K Mellon
}

Postgrad Med J 2004;80:320-327. doi: 10.1136/pgmj.2003.013508

For patients with urological cancers, immunotherapy is currently a treatment option for metastatic renal cell carcinoma, and those with "high risk" superficial bladder cancers. In this review, our current understanding of tumour immune escape is discussed. The principles and role of current immunotherapies for these tumours are described, and new areas of immunotherapeutic promise are highlighted.

See end of article for authors' affiliations

Correspondence to: Mr T R L Griffiths, Department of Cancer Studies and Molecular Medicine, University of Leicester, Clinical Sciences Unit, Leicester General Hospital, Gwendolen Road, Leicester LE5 4PW, UK; TRLG1@le.ac.uk

Submitted 7 August 2003 Accepted

3 November 2003
I n England and Wales, approximately 4000 new cases of renal cell carcinoma (RCC) are diagnosed each year; of these, one third exhibit metastatic disease at the time of initial presentation, and a third of the rest eventually develop distant metastases. For those presenting with metastatic disease, the median survival is approximately one year. ${ }^{1}$ Currently, cytoreductive nephrectomy followed by immunotherapy is advocated as the standard treatment for these patients, provided they have a good performance status. $^{2}{ }^{3}$ Patients with metastatic RCC and a solitary metastasis may also benefit from resection of the metastatic lesion. For patients with localised disease, open radical nephrectomy has been the treatment of choice, although the laparoscopic approach can now be offered in several centres. For small tumours $(<4 \mathrm{~cm})$, partial nephrectomy is an alternative option, particularly if the kidney is solitary, there is evidence of renal impairment, or the patient is predisposed to further renal tumours, such as those patients with von Hippel-Lindau disease. Patients subsequently presenting with metastatic disease could be offered immunotherapy; if the metastasis is solitary, the metastatic lesion could also be excised.

There are approximately 12000 new cases of bladder cancer each year in England and Wales. Over $90 \%$ are transitional cell carcinomas (TCCs). Of these, $50 \%$ are papillary and confined to the epithelium (Ta); $20 \%$ are papillary and invading the lamina propria $(\mathrm{Tl}) ; 5 \%$ are carcinoma in situ (Tis); $25 \%$ are solid and invading detrusor muscle (fig 1). It is the latter which account for the majority of deaths from bladder cancer. Within 24 hours of diagnostic transurethral resection, a single instillation of intravesical chemotherapy (mitomycin C or epirubicin) is administered. This has been shown to increase the recurrence-free interval. ${ }^{45}$ Patients with high risk superficial TCC are defined as those with Ta/ TlG3 TCC, Tis, and recurrent multifocal Ta/Tl TCC. If managed with transurethral resection alone, up to $60 \%$ of patients with Tis will subsequently progress to muscle-invasive or metastatic TCC. ${ }^{6}$ The standard treatment for these patients is to also receive an induction course of intravesical bacille Calmette-Guérin (BCG), followed by a maintenance regimen. ${ }^{7}$ These patients need regular cystoscopic follow up, under general anaesthetic initially. In contrast, in patients with solitary Gl/G2 "superficial" TCC, the main risk is tumour recurrence and not tumour progression. Up to $60 \%$ to $70 \%$ will recur with tumours of a similar stage and grade, which is usually not life threatening. For these patients, cystoscopic follow up only is sufficient; in most cases, flexible cystoscopy can be performed, thereby obviating the need for general anaesthesia. Patients with "high risk" superficial bladder cancer who recur with Tis or T1 or G3 TCC despite BCG are particularly vulnerable to progression. They are usually managed in the same way as those with apparently organ-confined muscle-invasive TCC of the bladder. The accepted treatments for the latter are radical cystectomy or radical radiotherapy. Despite this, only $40 \%$ of those with muscle-invasive TCC survive five years, because of subclinical metastases at presentation.

In this review, we describe the principles behind immune mediated cytotoxicity of tumours. The evidence base for the use of immunotherapy in the treatment of RCC and TCC of the bladder is discussed

\section{ANTIGEN PRESENTATION TO THE IMMUNE SYSTEM}

Defence against microbes is mediated by the early reactions of innate immunity, and the later responses of adaptive immunity. The defining characteristics of adaptive immunity are specificity and memory. The adaptive immune system responds to intracellular events in target cells by the recognition of protein fragments presented on the cell surface by transmembrane glycoproteins; the latter are called major histocompatibility complex (MHC) molecules. Antigens presented by MHC class I and II are recognised by the immune system. Complexes of MHC class

Abbreviations: BCG, bacille Calmette-Guérin; ECOG, Eastern Cooperative Oncology Group; EORTC-GU, European Organisation for Research and Treatment of Cancer Genitourinary Group; IFN- $\alpha$, interferon-alfa; IFN$\gamma$, interferon-gamma; IL, interleukin; MHC, major histocompatibility complex; NK, natural killer; NKT, natural killer T; RCC, renal cell carcinoma; SWOG', Southwest Oncology Group; TCC, transitional cell carcinoma 


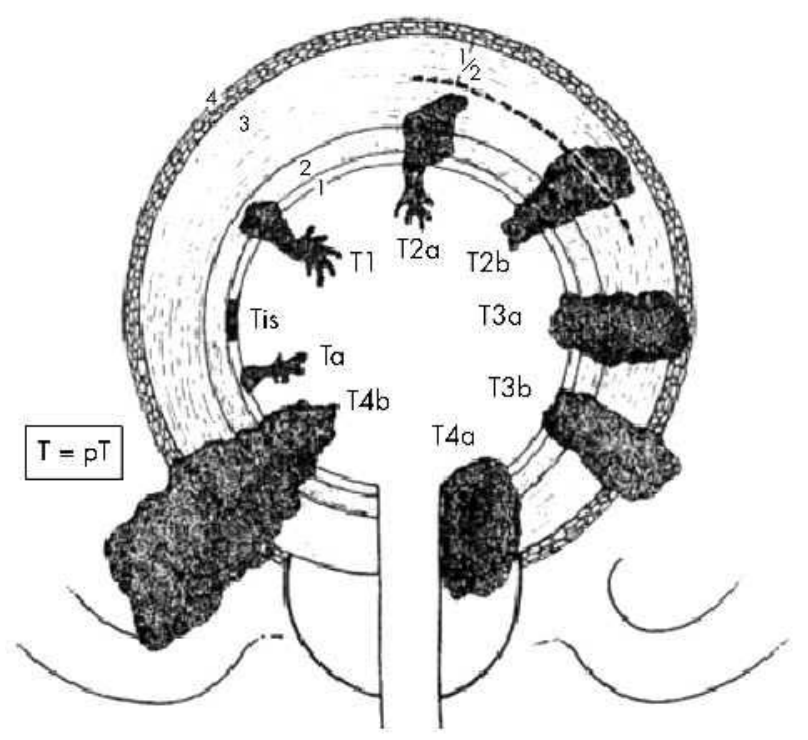

Figure 1 TNM (tumour, nodes, and metastasis) classification of bladder tumours, 1997. 1, epithelium; 2, lamina propria; 3, detrusor muscle; 4, perivesical fat.

I molecules and peptides derived from intracellular proteins are displayed on the surface of the majority of cells, and are recognised by $\mathrm{CD} 8$ positive $\mathrm{T}$ lymphocytes. In contrast, peptides presented by MHC class II molecules are derived from exogenous proteins; this complex is recognised by CD4 positive T lymphocytes. MHC class II molecules are mainly expressed on the surface of so-called professional antigen presenting cells, such as dendritic cells, macrophages, and activated B lymphocytes. Dendritic cells can interact with the adaptive immune systems via naïve CD4 positive and CD8 positive $\mathrm{T}$ cells, and the innate immune system via natural killer (NK) cells and natural killer T (NKT) cells.

\section{Role of effector cells}

CD4 positive $\mathrm{T}$ lymphocytes help to activate antigen presenting cells, and thereby maintain CD8 positive $\mathrm{T}$ cell mediated cytotoxicity. In contrast, NK cells are innate effectors, serving as a first line of immune defence. They characteristically lyse MHC mismatched cells, or cells having low levels or a lack of MHC expression. Unlike CD8 positive $\mathrm{T}$ cells, they lack peptide antigen specificity. Another type of effector, NKT cells, recognise a limited array of peptide and non-peptide (glycolipid) antigens presented by the non-polymorphic MHC-like molecule, CDl.

\section{Two signal model}

It has long been recognised that two signals are necessary for the initial activation of naïve $\mathrm{T}$ cells (fig 2 ). The two signal model $^{8}$ predicts that when an antigen is presented with a MHC molecule [signal 1], together with co-stimulatory molecules such as B7.1 (CD80) and B7.2 (CD86) [signal 2], an immune response will be generated. The second signal will usually happen only if the antigen is presented by a professional antigen presenting cell.

\section{Danger hypothesis}

The danger hypothesis focuses on the environment of antigen presentation and not the nature of the antigen; it therefore avoids the self versus non-self distinction. ${ }^{9}$ This hypothesis postulates that if an epitope is presented without a danger signal, it induces tolerance to that epitope; in contrast, if presented in the context of danger, it stimulates the immune system.

\section{CANCER AND IMMUNE ESCAPE MECHANISMS}

The failure of immune surveillance to prevent tumour development, and the observation that effective therapies can become ineffective over time, suggest that mechanisms exist, whereby tumours can escape the immune system.

Although most tumours produce peptides recognised by immune cells, many do not spontaneously induce a vigorous immune response. In the 1970s, Burnet suggested that the immune system had an important role in surveillance for transformed cells. ${ }^{10}$ An extension of this hypothesis is that the immune system selects for a more weakly immunogenic tumour, by clearing the immunogenic tumour cells. This may drive tumour cells towards a more malignant phenotype. ${ }^{11}$ Several possible mechanisms of immune escape have been proposed.

\section{Downregulation of MHC class I}

Downregulation of MHC class I by tumour cells enables evasion of recognition by CD8 positive T cells. For example, in bladder cancer, there is reduced expression of MHC class I compared with normal bladder mucosa. ${ }^{12}$ However, NK cells would still have the potential ability to kill cells that express low levels of class I MHC antigens. Tumour cells can also overcome this potential for lysis, by expression of just those specific MHC antigens that can engage inhibitory receptors, which prevent NK cell activation. ${ }^{13}$

\section{Fas-ligand mediated apoptosis of tumour infiltrating lymphocytes}

Expression of fas-ligand on tumour cells could mediate apoptosis of infiltrating lymphocytes by tumour cells. Indeed, both soluble and membrane-bound fas-ligand expression has been demonstrated in tumours. In vitro studies have demonstrated fas-ligand mediated apoptosis of $\mathrm{T}$ cells in bladder cancer. ${ }^{14}$

\section{Secretion of immunosuppressive cytokines}

This proposed method for tumour immune evasion is a nonspecific strategy, with broad consequences for the local immune system. Implicated secretory factors are transforming growth factor-beta, interleukin (IL)-10 and prostaglandin $\mathrm{E}_{2}$, which defend the tumour against tumour specific cytotoxic immune cells, and have been termed the tumour "firewall". ${ }^{15}$

\section{CANCER IMMUNOTHERAPY}

The fundamental assumption behind tumour immunotherapy is that under the appropriate conditions, malignant cells can be recognised as targets by the immune system. Immunotherapy can be active or passive.

\section{Active immunotherapy}

This is aimed at augmenting the weak host immune response to the tumours; strategies include use of cytokines and costimulators, tumour vaccines, and non-specific stimulation mediated by local administration of danger signals.

\section{Passive immunotherapy}

This includes the transfer of cultured immune effectors (termed adoptive immunotherapy) or antibodies with antitumour reactivity into patients. It is rapid, but does not lead to long lived immunity. Strategies of adoptive therapy include transfer of autologous lymphokine-activated killer cells and tumour-infiltrating lymphocytes expanded ex vivo.

Immunotherapateutic strategies in RCC and bladder cancer illustrate many of these principles.

\section{IMMUNOTHERAPY IN METASTATIC RENAL CELL CARCINOMA}

RCC is relatively resistant to radiotherapy, hormonal therapy, and chemotherapy. In a review of 30 chemotherapeutic 


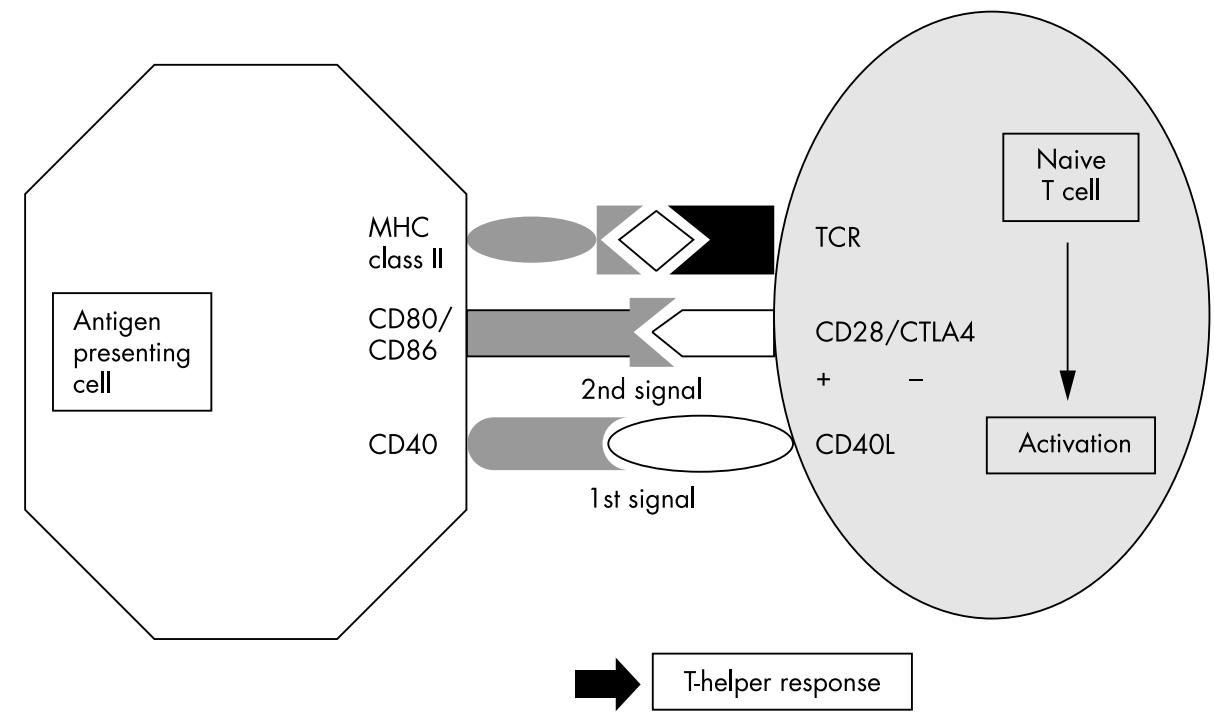

Figure 2 Antigen presentation to $\mathrm{T}$ cells $(\mathrm{TCR}=\mathrm{T}$ cell receptor).

agents tested in patients with advanced RCC between 1983 and 1993, the overall objective response rate was less than $9 \%{ }^{16}$ To date, no agent has been found to produce results that would justify its use as a single agent; metastatic RCC remains resistant to combination chemotherapy. It is possible that the relative chemoresistance of RCC is attributable to overexpression of the MDR-1 glycoprotein. ${ }^{17}$

\section{Active immunotherapy \\ Cytokine immunotherapy \\ Interferon-alfa}

Systemic cytokine immunotherapy for metastatic RCC began with the use of interferon-alfa (IFN- $\alpha$ ) in the early 1980s. IFN- $\alpha$ is a glycoprotein with antiviral and antitumour properties. Its mode of action in RCC is poorly understood, but it is probably a combination of stimulation of cell mediated cytotoxicity, direct antiproliferative activity, and an antiangiogenic effect. IFN- $\alpha$ also upregulates MHC class I antigen on tumour cells. A review of 1306 patients with metastatic RCC treated with a variety of IFN $-\alpha$ monotherapy regimens in phase I or II trials indicated an overall response rate of $13 \%$ and a complete response rate of $1.8 \%{ }^{18}$ The median duration of response was 6-10 months; these responses generally occurred in patients with pulmonary metastases.

Randomised clinical trials have compared IFN- $\alpha$ with other monotherapeutic approaches in metastatic RCC. In a pivotal multicentre randomised trial conducted by the Medical Research Council, patients with evaluable metastatic RCC and World Health Organisation performance status of 0 (no restriction) to 2 (ambulatory), were randomised to receive either subcutaneous IFN- $\alpha$ ( 10 MIU) three times a week for 12 weeks, or oral medroxyprogesterone $(300 \mathrm{mg}$ ) daily for the same duration. ${ }^{19}$ No limit on disease burden was

\section{Box 1: Immunotherapy for metastatic RCC}

- Only two agents have been shown to improve survival: IFN- $\alpha$ and IL-2.

- Debulking nephrectomy before IFN- $\alpha$ improves survival compared with IFN- $\alpha$ alone in patients with pulmonary metastases and good performance status. enforced. Those receiving IFN- $\alpha$ had a $28 \%$ reduction in the risk of death $(p=0.017)$. Their median survival was 8.5 months compared with 6 months in those who received medroxyprogesterone.

\section{Interleukin-2}

IL-2, a polypeptide lymphokine, was first described in 1976. The main secretory source is the T-helper cell. It is the principal stimulator of $\mathrm{T}$ cell growth; if antitumour $\mathrm{T}$ cells and NKT cells are present, they could be activated. Following encouraging in vitro and in vivo studies, clinical trials started in patients with metastatic RCC in 1984. A subsequent review of clinical results in 1714 patients with metastatic RCC treated with IL-2 monotherapy indicated an overall objective response rate of $15 \%{ }^{18}$

The optimal IL-2 regimen is not known. The most durable responses have been reported for high dose bolus intravenous IL-2 regimens. In one such series of highly selected patients with metastatic RCC, the complete response rate was 7\%, and three quarters of these remained in complete remission for over 40 months. ${ }^{20}$ However, high dose bolus IL-2 monotherapy is associated with a dose dependent toxicity; it requires hospitalisation and often inotropic support. The toxicity is believed to result from increased capillary permeability leading primarily to fluid retention and hypotension. Patients may also suffer from renal, hepatic, and haematological side effects.

High dose continuous infusion intravenous IL-2 has not been directly compared with intravenous bolus therapy. However, the need for intensive care support seems to be less, without an apparent reduction in durability of remission. ${ }^{21}$ An ongoing randomised trial organised by the National Cancer Institute is comparing high dose intravenous bolus IL-2 with low dose intravenous bolus IL-2 or subcutaneous IL-2 in patients with measurable metastatic RCC. ${ }^{22}$ Initial overall response rates were $16 \%, 4 \%$, and $11 \%$ respectively. Longer term data are awaited.

\section{Interferon-gamma}

In a phase III randomised trial conducted by the Canadian Urologic Group, interferon-gamma (IFN- $\gamma$ ) had no value in the treatment of metastatic RCC. ${ }^{23}$ More than half had two or more metastatic sites, and two thirds had excellent performance status (Karnofsky scores of $90 \%$ or $100 \%$ ). Response rates to IFN- $\gamma$ and placebo were $4 \%$ and $6 \%$ respectively, and there was no significant difference in survival. 
Interferon-alpha/interleukin-2 combinations

In vivo observations indicated possible synergistic antitumour activity of IL-2 and IFN- $\alpha{ }^{24}$ This led to clinical studies examining their combined use. A review of 1411 patients with metastatic RCC receiving a combination of IL-2 and IFN- $\alpha$ in phase I and II trials indicated an overall objective response rate of $20 \%$ and a complete response rate of $4 \%{ }^{18}$ In a phase III multicentre randomised trial, 425 patients with metastatic RCC were treated with a continuous intravenous infusion of IL-2, or subcutaneous injections of IFN- $\alpha$, or a combination of IL-2 and IFN- $\alpha$; the overall response rates were $6.5 \%, 7.5 \%$, and $18.6 \%(\mathrm{p}=0.01)$ respectively. ${ }^{25}$ However, the higher response rate achieved with the combination did not translate into improved survival, and toxic effects were more common in patients receiving IFN- $\alpha$. Patients who required palliative nephrectomy were invited to enter the trial only after they had undergone the operation.

\section{Cytokine/5-fluorouracil combinations}

The rationale for this combination is that chemotherapy may enhance immunogenicity by causing cellular damage and release of tumour cell antigens. These are processed by IFN- $\alpha$ stimulated antigen presenting cells which, in turn, activate IL-2 stimulated cellular effectors. In RCC, Atzpodien et al first showed that increased response rates could be achieved in an outpatient setting by combining subcutaneous injections of IL-2 and IFN- $\alpha$ with intravenous 5 -fluorouracil. ${ }^{26}$ In this regimen, patients with metastatic RCC received IFN- $\alpha$ (6-9 MU/m $\mathrm{m}^{2}$ once to three times a week) for eight weeks, combined sequentially with IL-2 (5-20 MU/m² three times a week) for the first four weeks and 5-fluorouracil $\left(750 \mathrm{mg} / \mathrm{m}^{2}\right.$ weekly) for the second four weeks. In another study, the same group randomised 78 patients with metastatic RCC who had undergone radical nephrectomy to receive either a similar regimen or oral tamoxifen in an outpatient setting. ${ }^{27}$ This required selection of patients with good or excellent performance status (Karnofsky performance status $>80 \%$ ); patients with brain metastases were excluded. The response rate for chemoimmunotherapy was 39\%; no objective responses were seen in the tamoxifen treated patients. The median overall survival in patients treated with chemoimmunotherapy was 24 months compared with 13 months in those treated with tamoxifen $(p=0.03)$. However, other centres have not been able to reproduce these results. In a review of 433 patients with metastatic RCC receiving various schedules of these three agents, an overall response rate of approximately $20 \%$ was found. ${ }^{18}$ In another randomised phase III multicentre trial, 131 patients with metastatic RCC were treated for eight weeks either with subcutaneous IL-2 and IFN- $\alpha$ or with the combination of IL-2, IFN- $\alpha$ and 5 -fluorouracil, the latter delivered by continuous intravenous infusion on days $1-5$ for week 1 and $5 .{ }^{28}$ Patients with brain metastases were again excluded. Response rates were low, and the cytokine/5-fluorouracil combination showed no added benefit. However, the dose of IL-2 was lower than that used by Atzpodein's group. Clearly, current data are conflicting, and additional randomised studies are needed to resolve this issue.

\section{Cytokine/vinblastine combinations}

Given that prior experience with vinblastine had shown low activity in RCC, it could be considered that vinblastine is little more than placebo. In a study of 160 patients with locally advanced or metastatic RCC, a regimen combining subcutaneous IFN- $\alpha$ with intravenous vinblastine was compared with vinblastine alone. ${ }^{29}$ Patients were required to be younger than 75 years of age, have a Karnofsky performance status $>50 \%$, and a life expectancy greater than three months. Patients with brain metastases or other malignancies were excluded. Treatment was for 12 months, or until progression of disease. The IFN- $\alpha$ dose was 3 MU three times a week for week 1 , and $18 \mathrm{MU}$ three times a week for subsequent weeks. If $18 \mathrm{MU}$ was intolerable, the dose was reduced to $9 \mathrm{MU}$. The dose of vinblastine in both groups was $0.1 \mathrm{mg} / \mathrm{kg}$ every three weeks. Response rates were $16.5 \%$ and $2.5 \%$ respectively $(\mathrm{p}=0.0025)$, and median survivals were 15.8 and 8.8 months respectively $(\mathrm{p}=0.0049)$.

\section{Cytokine/retinoic acid combinations}

A phase III trial was conducted comparing combined 13-cisretinoic acid and IFN- $\alpha$ with IFN- $\alpha$ alone in patients with locally advanced or metastatic RCC. ${ }^{30}$ Although the combination arm had a longer duration of response ( $33 v 22$ months, $\mathrm{p}=0.03)$, median survival was comparable. Moreover, quality of life was compromised in the combination arm, especially during the first eight weeks of treatment.

\section{Cytokine/thalidomide combinations}

Clear cell renal carcinoma is a hypervascular tumour. As well as modulating the immune system, IFN- $\alpha$ also has antiangiogenic activity. The importance of its angiostatic properties in RCC has however been questioned. ${ }^{31}$ Another antiangiogenic agent is thalidomide, which acts to lower the concentration of angiogenic factors such as TNF- $\alpha$ and vascular endothelial growth factor. Oral thalidomide (100 mg nightly until disease progression or toxicity) has been shown to induce a partial response in three of 18 patients with metastatic RCC; a further three patients experienced stabilisation of their disease for up to six months. ${ }^{32}$ All patients had a Eastern Cooperative Oncology Group (ECOG) performance status of 0 to 2, and a life expectancy of at least three months. An ongoing phase III trial is the ECOG trial (E2898) which compares IFN- $\alpha$ plus thalidomide versus IFN- $\alpha$ alone in patients with untreated metastatic or unresectable RCC.

\section{Variants of interleukin-2 and interferon}

The rationale for developing these variants is to design cytokines with additional pharmacokinetic features, so as to reduce systemic toxicity. Strategies include increasing IL-2 locally within the tumour, increasing IL-2 selectivity for T cells rather than NK cells (which are a putative cause of IL-2 intolerance), or alteration of dosing frequency.

Leuvectin is a product in which a plasmid DNA expression vector coding for the human IL-2 protein is contained in a cationic lipid that promotes DNA transfection. IL-2 production therefore occurs locally within the tumour. Another variant of IL-2 called BAY 50-4798 is in early development. Through targeted mutagenesis, a synthetic form of IL-2 has been developed that has approximately 3000 -fold selectivity for T cells compared with NK cells. A variant of IFN- $\alpha$ has also been developed called pegylated interferon. The once-aweek dosing schedule associated with pegylated interferon may provide longer sustained IFN- $\alpha$ levels.

\section{Nephrectomy/cytokine immunotherapy combinations}

Before the era of immunotherapy, one year and three year survival rates for patients with metastatic RCC were $26 \%$ and $4 \%$ respectively. ${ }^{1}$ In these circumstances, the natural history was not improved by debulking nephrectomy. ${ }^{13}$ Despite this, nephrectomy did have an unquestioned role in palliation of symptoms, in patients with a good performance status. ${ }^{34}$ It was recommended for patients with bleeding, pain, or hypercalcaemia to provide improvement in their quality of life.

With the advent of modern immunotherapy, the role of nephrectomy has once again been the subject of much debate. The publication of two prospective randomised phase III trials has swung the pendulum in favour of early nephrectomy for patients with a good performance status. ${ }^{23}$ Both trials compared nephrectomy plus subcutaneous IFN- $\alpha$ 
with IFN- $\alpha$ alone in patients with metastatic RCC outside the region to be resected. In both trials, patients were excluded if they had received prior treatment with chemotherapy or biological response modifiers. The European Organisation for Research and Treatment of Cancer Genitourinary Group (EORTC-GU) trial 30947 reported a 10 month median overall survival advantage in the combination group compared with the IFN- $\alpha$ alone group (17 $\vee 7$ months; $\mathrm{p}=0.03) .^{2}$ In this study, patients with evidence of brain metastases were excluded. The Southwest Oncology Group (SWOG) trial 8949 noted a three month median overall survival advantage for the combination group ( $11.1 v 8.1$ months; $\mathrm{p}=0.02){ }^{3}$ Patients with only lung metastases benefited more from nephrectomy than those with other metastases $(p=0.008)$.

Further support for cytoreductive nephrectomy followed by immunotherapy comes from a retrospective analysis of 89 patients with metastatic RCC who met the eligibility criteria for the SWOG study, and who received IL-2 based regimens after nephrectomy. ${ }^{35}$ The median survival in these patients was 16 months; one fifth survived five years.

\section{Passive (adoptive) immunotherapy}

Lymphokine activated killer cells

NK cells can be activated ex vivo by IL-2 to form lymphokineactivated killer cells. One strategy is to expand these cells ex vivo using high dose IL-2, and to then reinfuse them usually in conjunction with IL-2, back into the patient. Following studies demonstrating enhanced in vitro cytotoxicity of RCC cells, clinical trials started. In one phase III trial for patients with metastatic RCC, high dose IL-2 alone was compared with high dose IL-2 in combination with lymphokineactivated killer cells. ${ }^{36}$ There was no difference in response rates between the two groups. Consequently, this strategy has largely been abandoned as a treatment for these patients.

Tumour-infiltrating lymphocytes

Another strategy is to harvest the subset of lymphocytes which infiltrate tumour biopsies. The rationale is that they represent clones, which at least target the correct location. These lymphocytes, called tumour-infiltrating lymphocytes can then undergo IL-2 mediated expansion ex vivo. They are then transferred back to the patient. Again, in vitro studies suggested that this subpopulation of CD8 positive lymphocytes was more cytotoxic to RCC than the general population of CD8 positive lymphocytes. On this basis, a multicentre phase III trial was conducted, to compare the antitumour activity of a combination of CD8 positive tumour-infiltrating lymphocytes and low dose IL-2 with IL-2 alone, after radical nephrectomy in patients with metastatic RCC. ${ }^{37}$ The combination failed to improve response rates, and one year survival rates were similar.

\section{Future strategies}

Tumour vaccines

A variety of approaches are currently undergoing trial in RCC, but none have shown convincing benefit to date. First generation tumour vaccines include autologous cancer cells or lysates harvested from patients, and then returned to them together with non-specific adjuvants. Second generation tumour vaccines employ genetically modified tumour cells, dendritic cells, and recombinant tumour antigens.

The procurement of heat shock protein-tumour antigen vaccines or induction of heat shock protein expression is an exciting prospect. Heat shock proteins are intracellular molecules that act as chaperones for antigens, and may stimulate an immune response. Several tumour specific cytotoxic $\mathrm{T}$ lymphocytes and dendritic cells can recognise members of the heat shock protein family. Therefore heat shock protein complexes released by necrotic cells function as endogenous danger signals as well as a method to cross present antigens by dendritic cells. Of interest, in a small study of 43 patients with metastatic RCC, low expression of heat shock protein 70 correlated with early relapse. ${ }^{38}$

\section{Allogeneic stem cell transplant}

Allogeneic stem cell transplants have been used for decades in the treatment of haematological malignancies. It was assumed that its main role was to rescue the recipient from myeloablation caused by high dose chemotherapy. However, it is likely that the immune mediated rejection of malignancy by donor $\mathrm{T}$ cells in the allograft may be more important. A study of non-myeloablative conditioning medium of fluarabine and cyclophosphamide in 19 patients with metastatic RCC was recently reported. ${ }^{39}$ In this trial, 10 patients had regression of metastases; three of these patients achieved complete remissions that lasted more than one year.

\section{IMMUNOTHERAPY IN BLADDER CANCER Intravesical BCG \\ History}

During development of an antituberculous vaccine at the Pasteur Institute in Paris in 1908, Calmette and Guérin observed that a highly virulent strain of bovine tubercle bacillus gradually lost its virulence when repeatedly cultured. As a result, the BCG vaccine was established, which consists of live attenuated Mycobacterium bovis. The use of BCG as a treatment for cancer was first investigated following an important observation by Pearl in 1929; a reduced incidence of malignancy was reported in autopsies of tuberculosis sufferers compared with a control group. ${ }^{40}$ Following this, BCG was tested in a number of tumour models including malignant melanoma, adenocarcinoma of the kidney, and head and neck cancers. In 1974, the conditions required to establish an effective antitumour response were formulated; these included an ability to develop an immune response to mycobacteria antigens, adequate number of living bacilli, close contact between BCG and tumour cells, and a small tumour burden. ${ }^{41}$

Clearly, instillation of BCG into bladders with superficial cancer was an attractive option. Indeed, histopathological examination of canine bladders after intracavitary BCG showed inflammatory cellular infiltrates. ${ }^{42}$ This culminated in the first study using intravesical BCG for the treatment of patients with superficial bladder cancer in $1976 .{ }^{43}$ The original paper describes intravesical treatment of nine patients with superficial bladder cancer, and supplemented with intradermal inoculation. Treatments were repeated weekly for six weeks.

\section{$B C G$ and route of administration}

An induction schedule of weekly intravesical instillations for six weeks is still continued in current practice. Currently, a further maintenance regimen is recommended as discussed

Box 2: Immunotherapy for "high risk" superficial bladder cancer

- The optimal schedule and dose of BCG is unknown.

- Intravesical instillation is the optimal route of administration of BCG.

- Maintenance intravesical BCG:

- Is superior to a standard induction course in preventing recurrence.

- Delays and may prevent progression to muscleinvasive or metastatic TCC. 
below. In the UK, the intradermal component has been abandoned. In a randomised trial, clinical efficacy after intravesical instillation alone was similar to that after a combination of intravesical instillations plus percutaneous administration in patients, with recurrent superficial bladder cancer. ${ }^{44}$

\section{Mechanism of action}

To date, the precise mechanism of action of intravesical BCG remains unknown. The mycobacteria are thought to bind to the bladder wall via the interaction between the bacterial antigen 85 complex and fibronectin. ${ }^{45}$ A likely scenario is that exposure to BCG acts as a danger signal activating local dendritic cells. ${ }^{46}$ The activated dendritic cells may then migrate to local lymph nodes where peptides of BCG and TCC origin are presented to T lymphocytes. The differentiation of naïve CD4 positive $\mathrm{T}$ cells into the T-helper type 1 (Thl) subset is indispensable for successful treatment of superficial bladder cancer with BCG. $^{47}$ The activated $\mathrm{T}$ lymphocytes then migrate to the urothelium and lyse TCC cells, either directly through the CD8 positive population or indirectly by activating NK cells.

\section{Intravesical therapy and recurrence}

Many trials have compared intravesical BCG to chemotherapy. Ultimately, the choice of treatment in patients will depend upon the patient's risk of recurrence and progression. In papillary tumours at low risk of recurrence, intravesical BCG and mitomycin $\mathrm{C}$ are equivalent. ${ }^{48}$ Since BCG has a greater risk of toxicity, intravesical chemotherapy is favoured in these patients. In those with recurrent multifocal Ta/T1 TCC without Tis, BCG is superior to mitomycin $\mathrm{C}^{49}$ in preventing recurrence. Sequential mitomycin $\mathrm{C}$ and BCG has also been assessed, but does not have more efficacy than mitomycin $\mathrm{C}$ alone. ${ }^{50}$ With regard to the treatment of Tis, BCG is the recommended option; in a meta-analysis, the complete response to BCG $(70 \%)$ is superior to that of mitomycin C $(40 \%){ }^{51}$

In patients with Tis and others at high risk of recurrence, the SWOG introduced a three year maintenance intravesical and percutaneous BCG regimen comprising a total of 27 instillations. They showed that this regimen was superior to a standard induction course, in terms of recurrence-free survival. ${ }^{52}$ However, there was no increase in overall survival, and only $16 \%$ of patients completed the regimen.

\section{Intravesical therapy and progression}

A meta-analysis comprising over 2500 patients showed that intravesical chemotherapy increases the recurrence-free interval when compared with transurethral resection alone, but it did not affect the time to progression to muscleinvasive disease. ${ }^{53}$ Until recently, controversy existed whether BCG could delay or prevent progression. There is no single randomised controlled trial with unequivocal proof that BCG prevents tumour progression, but individual studies have had only limited power. Certainly, in retrospective studies of patients at high risk of progression, the medium term results following a single induction course of BCG are disappointing. Indeed, in patients with Tis concomitant with Tl TCC, only $38 \%$ of complete responders were alive with bladder intact at five years. ${ }^{54}$ A recent pivotal meta-analysis of 24 randomised trials carried out by the EORTC has shown that maintenance BCG but not a single induction course of BCG prevents or delays progression. ${ }^{7}$ A $37 \%$ reduction in the odds of progression was observed for the 20 trials in which a maintenance BCG regimen was given $(p=0.00004)$.

\section{Optimal BCG regimen}

Clearly, a maintenance schedule of BCG is needed for optimal efficacy, but the precise regimen to achieve this remains unknown. Many different schedules have been employed, ranging from a total of 10 instillations given during 18 weeks to 30 instillations given over three years. Both the EORTC and the SWOG have given 27 instillations over three years in their trials.

\section{Optimal dose of BCG}

The optimal dose remains unknown. A number of trials have compared the efficacy and toxicity of reduced doses of BCG with full doses. One of the larger studies, compared one third dose with full dose BCG. ${ }^{55}$ In the reduced dose schedule, efficacy was not compromised, fewer patients reported toxicity, but the incidence of severe systemic toxicity was not reduced.

\section{Optimal strain of BCG}

Although differences may exist between strains, there is currently no evidence to suggest that they differ in clinical efficacy. In the BCG progression meta-analysis, five different strains were included: Tice, Connaught, Pasteur, RIVM, and A Frappier. ${ }^{7}$ Their ability to prevent progression was similar.

\section{Optimal time point for assessment of response to BCG}

Recent data suggest that evaluating response to intravesical BCG is best assessed at six months, whether or not additional maintenance instillations are administered. ${ }^{56}$ The purpose of the three month check cystoscopy is to exclude early progression to muscle-invasive TCC. Further support for this concept can be found in the SWOG maintenance trial; $60 \%$ of patients with residual Tis at three months' follow up were disease-free after $6+3$ instillations of BCG. ${ }^{52}$

\section{BCG toxicity}

Low grade fever $\left(<38.5^{\circ} \mathrm{C}\right)$ and cystitis are common side effects of BCG, and usually subside within 48 hours. Allergic reactions to $\mathrm{BCG}$ are uncommon $(<1 \%$ of cases); they include arthritis and skin rashes. Haematogenous spread of BCG is responsible for systemic complications. The most serious complication is generalised "BCGitis" which requires urgent treatment with four agent antituberculous therapy. These patients usually have a high fever $\left(>38.5^{\circ} \mathrm{C}\right)$ with impaired haemodynamic status. Systemic complications are rare provided certain precautions are followed. Intravesical BCG should not be given within 14 days of transurethral resection if there is an active urinary tract infection, frank haematuria, traumatic catheterisation, or immunodeficiency. Co-administration of prophylactic isoniazid does not reduce toxicity. ${ }^{57}$

\section{Mycobacterial cell wall extract}

Mycobacterial cell wall extracts have been investigated as an alternative to BCG; the potential advantage is that administration of a live attenuated organism is avoided, and therefore toxicity could be minimised. Early results in patients with Tis have confirmed that toxicity is less following instillation of mycobacterial cell wall extracts compared with live BCG, but efficacy is also less. ${ }^{58}$

\section{Recombinant BCG}

In a small study, combining intravesical low dose BCG plus IFN- $\alpha$ appeared to be effective in many cases of high risk superficial TCC previously deemed to be BCG refractory. ${ }^{59}$ BCG which has been genetically engineered to secrete recombinant human IFN- $\alpha$ has demonstrated enhanced immunogenicity compared with BCG. ${ }^{60}$

\section{CONCLUSIONS}

The use of biological therapies has an established role in the treatment of metastatic RCC and "high risk" superficial 


\section{Box 3: Key references}

- Mickisch GH, Garin A, van Poppel H, et al. Radical nephrectomy plus interferon-alfa-based immunotherapy compared with interferon alfa alone in metastatic renal-cell carcinoma: a randomised trial. Lancet $2001 ; 358: 966-70$.

- Flanigan RC, Salmon SE, Blumenstein BA, et al. Nephrectomy followed by interferon alfa-2b compared with interferon alfa- $2 \mathrm{~b}$ alone for metastatic renal-cell cancer. N Engl J Med 2001;345:1655-9.

- Atzpodien J, Kirchner H, Hanninen EL, et al. IL-2 in combination with IFN-alpha and 5-FU versus tamoxifen in metastatic renal cell carcinoma: long-term results of a controlled randomised clinical trial. $\mathrm{Br} J$ Cancer 2001;85:1130-6.

- Sylvester RJ, van der Meijden AP, Lamm DL, et al. Intravesical bacillus Calmette-Guerin reduces the risk of progression in patients with superficial bladder cancer: a meta-analysis of the published results of randomised clinical trials. J Urol 2002;168;1964-70.

- Lamm D, et al. Maintenance bacillus Calmette-Guerin immunotherapy for recurrent Ta, T1 and carcinoma in situ transitional cell carcinoma of the bladder: a randomised Southwest Oncology study. J Urol 2000; 163:1124-9.

bladder cancer. Despite this, there is a paucity of long term survivors in metastatic RCC. In superficial bladder cancer, intravesical maintenance BCG has been shown to delay progression to muscle-invasive or metastatic TCC, but it remains controversial whether it prevents progression in the long term. New biological therapies are being assessed in both tumours.

\section{QUESTIONS (ANSWERS AFTER REFERENCES)}

\section{Q1. Which of the following statements are true?}

(A) Downregulation of MHC class I expression enables evasion of recognition by CD4 positive cells

(B) NK cells can lyse cells with low levels of MHC class I expression

(C) IFN- $\alpha$ upregulates MHC class I expression

(D) NK cells are part of the adaptive immune system

(E) Active immunotherapy includes the transfer of cultured tissue infiltrating lymphocytes

Q2. In patients with newly diagnosed metastatic RCC:

(A) Median survival is approximately two years

(B) Combination chemotherapy is a successful option

(C) Cytoreductive nephrectomy followed by immunotherapy should be considered if there are pulmonary metastases and performance status is good

(D) IFN- $\alpha$ has shown a modest survival benefit in phase III trials comparing IFN- $\alpha$ with vinblastine or medroxyprogesterone

(E) Combined immunotherapy and 5-fluorouracil has shown promise

Q3. IL-2:

(A) Is the principal stimulator of $\mathrm{T}$ cell growth

(B) Is mainly secreted by CD8 positive T cells
(C) Monotherapy in metastatic RCC has a 30\% response rate

(D) Immunotherapy causes equal toxicity irrespective of intravenous or subcutaneous administration

(E) Is a polypeptide

\section{Q4. Intravesical BCG:}

(A) Is a live attenuated vaccine

(B) Is effective if there is a large tumour burden

(C) Is the treatment of choice for Tis

(D) Should be instilled within 24 hours of transurethral resection of bladder tumour

(E) Is superior to chemotherapy in preventing recurrence

\section{Q5. An induction course of intravesical BCG for superficial bladder cancer:}
(A) Classically consists of six instillations
(B) Has been shown to delay progression
(C) Has been shown to improve survival
(D) Should be co-administered with intradermal BCG
(E) Should be given to patients with solitary TaGl TCC

\section{Q6. Following intravesical BCG:}

(A) Low grade fever and cystitis are common side effects

(B) Generalised BCGitis should be considered if the patient has a fever of $37.5^{\circ} \mathrm{C}$

(C) Generalised BCGitis requires urgent antituberculous therapy

(D) Allergic complications occur in 5\% of cases

(E) Prophylactic isoniazid prevents complications

\section{Q7. Which of the following statements are true?}

(A) In superficial bladder cancer, a complete response following intravesical BCG is associated with a T-helper 2 immune response

(B) Heat shock proteins are intracellular molecules that act as chaperones for antigens

(C) Treatment of metastatic RCC with a combination of IFN- $\alpha$ and IL- 2 is associated with better survival than treatment with IFN- $\alpha$ alone

(D) Dendritic cells are professional antigen presenting cells

(E) The aim of active immunotherapy is to augment the host's immune response to tumours

\section{Authors' affiliations}

T R L Griffiths, J K Mellon, Department of Cancer Studies and Molecular Medicine, University of Leicester, Leicester General Hospital, Leicester, UK

\section{REFERENCES}

1 Patel NP, Lavengood RW. Renal cell carcinoma: natural history and results of treatment. J Urol 1978;119:722-6.

2 Mickisch GH, Garin A, van Poppel H, et al. European Organisation for Research and Treatment of Cancer (EORTC) Genitourinary Group. Radical nephrectomy plus interferon-alfa-based immunotherapy compared with interferon-alfa alone in metastatic renal-cell carcinoma: a randomised trial. Lancet 2001;358:966-70.

3 Flanigan RC, Salmon SE, Blumenstein BA, et al. Nephrectomy followed by interferon alfa- $2 b$ compared with interferon alfa- $2 b$ alone for metastatic renalcell cancer. N Engl J Med 2001;345:1655-9.

4 Oosterlinck W, Kurth KH, Schroder F, et al. A prospective European Organisation for Research and Treatment of Cancer Genitourinary Group randomised trial comparing transurethral resection followed by a single 
intravesical instillation of epirubicin or water in a single stage $\mathrm{Tl}$ papillary carcinoma of bladder. J Urol 1993;149:749-52.

5 Tolley DA, Parmar MK, Grigor KM, et al. The effect of intravesical mitomycin C on recurrence of newly diagnosed superficial bladder cancer: a further report with 7 years of follow up. J Urol 1996:155:1233-8.

$6 \mathrm{Utz}$ DC, Hanash KA, Farrow GM. The plight of the patient with carcinoma in situ of the bladder. J Urol 1970;111:160-4.

7 Sylvester RJ, van der Meijden AP, Lamm DL. Intravesical bacillus CalmetteGuerin reduces the risk of progression in patients with superficial bladder cancer: a meta-analysis of the published results of randomised clinical trials. J Urol 2002;168:1964-70.

8 Lafferty KJ, Cunningham AJ. A new analysis of allogeneic interactions. Aust J Exp Biol Med Sci 1975;53:27-42.

9 Matzinger P. The danger model: a renewed sense of self. Science 2002;296:301-5.

10 Burnet FM. Immunological surveillance in neoplasia. Transplant Rev $1971 ; 7: 3-25$

11 Semour K, Pettis S, O'Flaherty E, et al. Selection of metastatic tumour phenotypes by host immune systems. Lancet 1999;354:1989-91.

12 Levin I, Klein T, Golstein J, et al. Expression of class I histocompatibility antigens in transitional cell carcinoma of the urinary bladder in relation to survival. Cancer 1991;68:2591-4.

13 Keating PJ, Cromme FV, Duggan-Keen M, et al. Frequency of downregulation of individual HLA-A and -B alleles in cervical carcinomas in relation to TAP-1 expression. Br J Cancer 1995;72:405-11.

14 Pettit SJ, Ali S, O'Flaherty E, et al. Bladder cancer immunogenicity: expression of $C D 80$ and $C D 86$ is insufficient to allow primary $C D+T$ cell activation in vitro. Clin Exp Immunol 1999;1 16:48-56.

15 Shah AH, Lee C. TGF-beta-based immunotherapy for cancer: breaching the tumour firewall. Prostate 2000;45:167-72.

16 Yagoda A, Abi-Rached B, Petrylak D. Chemotherapy for advanced renal-cell carcinoma: 1983 to 1993. Semin Oncol 1995;22:42-60.

17 Goldstein L, Galski H, Fojo A, et al. Expression of a multi-drug resistance gene in human tumours (abstract). Proc Am Assoc Cancer Res 1988;29:298.

18 Bukowski RM. Immunotherapy in renal cell carcinoma. Oncology 1999;13:801-10.

19 Medical Research Council Renal Cancer Collaborators. Interferon-alpha and survival in metastatic renal carcinoma: early results of a randomised controlled trial. Lancet 1999;353:14-17.

20 Fisher RI, Rosenberg SA, Fyfe G. Long-erm survival update for high-dose recombinant interleukin-2 in patients with renal cell carcinoma. Cancer J Sci Am 2000;6(suppl):S55-7.

21 Gold PJ, Thompson JA, Markowitz DR, et al. Metastatic renal cell carcinoma: long-term survival after therapy with high-dose continuous-infusion interleukin-2. Cancer J Sci Am 1997;3(suppl 1):S85-91.

22 Yang JC, Rosenberg SA. An ongoing prospective randomised comparison of interleukin-2 regimens for the treatment of metastatic renal cell cancer. Cancer J Sci Am 1997;3(suppl 1):S79-84.

23 Gleave ME, Elhilali $M$, Fradet $Y$, et al. Interferon gamma-1b compared with placebo in metastatic renal-cell carcinoma. Canadian Urologic Oncology Group. N Engl J Med 1998;338:1265-71.

24 Chikkala NF, Lewis I, Ulchaker J, et al. Interactive effects of alpha-interferon $\mathrm{A} / \mathrm{D}$ and interleukin 2 on murine lymphokine activayed killer activity: analysis at the effector and precursor level. Cancer Res 1990;50:1176-82.

25 Negrier S, Escudier B, Lasset C, et al. Recombinant human interleukin-2, recombinant human interferon alfa-2a, or both in metastatic renal-cell carcinoma. Group Francais d'Immunotheapie. N Engl J Med 1998;338:1272-8.

26 Atzpodien J, Kirchner $\mathrm{H}$, Hanninen EL, et al. Interleukin-2 in combination with interferon-alpha and 5 -fluorouracil for metastatic renal cell carcinoma. Eur J Cancer 1993;29A(suppl 5):S6-8.

27 Atzpodien J, Kirchner $\mathrm{H}$, Illiger HJ, et al. IL-2 in combination with IFN-alpha and 5 -FU versus tamoxifen in metastatic renal cell carcinoma: long-term results of a controlled randomised clinical trial. Br J Cancer 2001;85:1130-6

28 Negrier S, Caty A, Lesinple T, et al. Treatment of patients with metastatic renal carcinoma with a combination of subcutaneous interleukin-2 and interferon alfa with or without fluorouracil. Groupe Francais d'Immunotherapie, Federation Nationale des Centres de Lutte Contre le Cancer. J Clin Oncol 2000;18:4009-15.

29 Pyrhonen S, Salminen E, Ruutu M, et al. Prospective randomised trial of interferon alfa-2a plus vinblastine versus vinblastine alone in patients with advanced renal cell cancer. J Clin Oncol 1999;17:2859-67.

30 Motzer RJ, Murphy BA, Bacik J, et al. Phase III trial of alfa-2a with or without 13-cis retinoic acid for patients with advanced renal cell carcinoma. J Clin Oncol 2000; 18:2972-80.

31 Sasamura H, Takahashi A, Miyao A, et al. Inhibitory effect on expression of angiogenic factors by antiangiogenic agents in renal cell carcinoma. $\mathrm{Br} J$ Cancer 2002;86:768-73.

32 Eisen T, Boshoff C, Mak I, et al. Continuous low dose thalidomide: a phase II study in advanced melanoma, renal cell, ovarian and breast cancer. Br J Cancer 2000;82:812-7.

33 Dekernion JB, Ramming KP, Smith RB. The natural history of metastatic renal cell carcinoma: a computer analysis. J Urol 1978;120:148-52.

34 Motzer RJ, Bander NH, Nanus DM. Renal-cell carcinoma. N Engl J Med 1996:335:865-75.

35 Pantuck AJ, Belldegrun AS, Figlin RA. Nephrectomy and interleukin-2 for metastatic renal-cell carcinoma. N Engl J Med 2001;345:171 1-2.
36 Rosenberg SA, Lotze MT, Yang JC, et al. Prospective randomised trial of highdose interleukin-2 alone or in conjunction with lymphokine-activated killer cells for the treatment of patients with advanced cancer. J Natl Cancer Inst 1993;85:622-32

37 Figlin RA, Thompson JA, Bukowski RM, et al. Multicenter, randomised, phase III trial of $\mathrm{CD} 8(+)$ tumour-infiltrating lymphocytes in combination with recombinant interleukin-2 in metastatic renal cell carcinoma. J Clin Oncol 1999;17:2521-9.

38 Santarosa M, Favaro D, Quaia M, et al. Expression of heat shock protein 72 in renal cell carcinoma: possible role and prognostic implications in cancer patients. Eur J Cancer 1997;33:873-7.

39 Childs R, Chernoff A, Contentin N, et al. Regression of metastatic renal-cell carcinoma after nonmyeloablative allogeneic peripheral-blood stem-cell transplantation. N Engl J Med 2000;343:750-8.

40 Pearl R. Cancer and tuberculosis. Am J Hyg 1929;9:97

41 Zbar B, Rapp HJ. Immunotherapy of guinea pig cancer with BCG. Cancer 1974;34:1532-40.

42 Bloomberg SD, Brosman SA, Hausman MS, et al. The effects of BCG on the dog bladder. Invest Urol 1975;12:423-7.

43 Morales A, Eidinger D, Bruce AW. Intracavitary bacillus Calmette-Guerin in the treatment of superficial bladder tumours. J Urol 1976;116:180-3.

44 Luftenegger W, Ackermann DK, Futterlieb A, et al. Intravesical versus intravesical plus intradermal bacillus Calmette-Guerin: a prospective randomised study in patients with recurrent superficial bladder tumours. J Urol 1996; 155:483-7.

45 Zlotta AR, Drowart A, Van Vooren JP, et al. Evolution and clinical significance of the T cell proliferative and cytokine response directed against the fibronectin binding antigen 85 complex of bacillus Calmette Guerin during intravesical treatment of superficial bladder cancer. J Urol 1997;157:492-8.

46 Matzinger P. An innate sense of danger. Semin Immunol 1998;10:399-415.

47 Saint F, Patard JJ, Maille P, et al. T helper $1 / 2$ lymphocyte urinary cytokine profiles in responding and nonresponding patients after 1 and 2 courses of bacillus Calmette-Guerin for superficial bladder cancer. J Uro $2001 ; 166: 2142-7$

48 Vegt PD, Witjes JA, Witjes WP, et al. A randomised study of intravesical mitomycin C, bacillus Calmette-Guerin Tice and bacillus Calmette-Guerin RIVM treatment in pTa-pT1 papillary carcinoma and carcinoma in situ of the bladder. J Urol 1995; 153:929-33.

49 Lamm DL, van der Meijden AP, Akaza H, et al. Intravesical chemotherapy and immunotherapy: how do we assess their effectiveness, and what are their limitations and uses? Int J Urol 1995;2(suppl 2):23-35.

50 Witjes JA, Caris CT, Mungan NA, et al. Results of a randomised phase III trial of sequential intravesical therapy with mitomycin $\mathrm{C}$ and bacillus CalmetteGuerin versus mitomycin $C$ alone in patients with superficial bladder cancer. $J$ Urol 1998; 160:1668-71

51 Bouffioux C. Intravesical adjuvant treatment in superficial bladder cancer. A review of the question after 15 years of experience with the EORTC GU group. Scand J Urol Nephrol Suppl 1991;138:167-77.

52 Lamm DL, Blumenstein BA, Crissman JD, et al. Maintenance bacillus CalmetteGuerin immunotherapy for recurrent $\mathrm{Ta}, \mathrm{Tl}$ and carcinoma in situ transitional cell carcinoma of the bladder: a randomised Southwest Oncology study. J Urol 2000;163:1124-9.

53 Pawinski A, Sylvester R, Kurth KH, et al. A combined analysis of European Organisation for Research and Treatment of Cancer, and Medical Research Council randomised clinical trials for the prophylactic treatment of stage TaT 1 bladder cancer. European Organisation for Research and Treatment of Cancer Genitourinary Tract Cancer Cooperative Group, and the Medical Research Council Working Party on Superficial Bladder Cancer. J Urol 1996; 156:1934-40

54 Griffiths TR, Charlton M, Neal DE, et al. Treatment of carcinoma in situ with intravesical bacillus Calmette-Guerin without maintenance. J Urol 2002;167:2408-12

55 Martinez Pineiro JA, Flores N, Isorna S, et al. Long-term follow-up of a randomised prospective trial comparing a standard $81 \mathrm{mg}$ dose of intravesical bacillus Calmette-Guerin with a reduced dose of $27 \mathrm{mg}$ in superficial bladder cancer. BJU Int 2002;89:671-80.

56 Herr HW, Dalbagni G. Defining bacillus Calmette-Guerin refractory superficial bladder tumours. J Urol 2003;169:1706-8.

57 Vegt PD, van der Meijden AP, Sylvester R, et al. Does isoniazid reduce side effects of intravesical bacillus Calmette-Guerin therapy in superficial bladder cancer? Interim results of European Organisation for Research and Treatment of Cancer Protocol 30911. J Urol 1997;157:1246-9.

58 Morales A, Chin JL, Ramsey EW. Mycobacterial cell wall extract for treatment of carcinoma in situ of the bladder. J Urol 2001;166:1633-7.

59 O'Donnell MA, Krohn J, DeWolf WC. Salvage intravesical therapy with interferon-alpha $2 \mathrm{~b}$ plus low dose bacillus Calmette-Guerin is effective in patients with superficial bladder cancer in whom bacillus Calmette-Guerin alone previously failed. J Urol 2001;166:1300-4.

60 Luo Y, Chen X, Han R, et al. Recombinant bacille Calmette-Guerin (BCG) expressing human interferon-alpha $2 B$ demonstrates enhanced immunogenicity. Clin Exp Immunol 2001;123:264-70.

\section{ANSWERS (TRUE)}

Q1. B, C; Q2. C, D, E; Q3. A, E; Q4. A, C, E; Q5. A; Q6. A, C; Q7. B. D, E. 